\title{
Properties of B and P doped Ge nanowires
}

\author{
H. Peelaers, ${ }^{\text {a) }}$ B. Partoens, ${ }^{\text {b) }}$ and F. M. Peeters ${ }^{\text {c) }}$ \\ Departement Fysica, Universiteit Antwerpen, Groenenborgerlaan 171, B-2020 Antwerpen, Belgium
}

(Received 9 May 2007; accepted 2 June 2007; published online 25 June 2007)

\begin{abstract}
An $a b$ initio study of the formation and segregation energies of $\mathrm{B}$ and $\mathrm{P}$ doped $\mathrm{Ge}$ nanowires oriented along the [110] direction is performed for fully relaxed H-passivated nanowires. The authors found the preferential dopant positions and the associated formation energies. Edge positions are favorable positions for both types of dopants. In the presence of dangling bond defects $\mathrm{P}$ dopants will highly favor the edge positions near the dangling bond, thereby trapping the additional carrier. The results are compared with those found for doped Si nanowires. (C) 2007 American Institute of Physics. [DOI: 10.1063/1.2752107]
\end{abstract}

Nanoscale structures such as nanowires (NWs) have generated a lot of scientific interest lately as possible building blocks for nanoscale devices. Germanium NWs are particularly interesting because of their compatibility with $\mathrm{Si}$ and their higher carrier mobilities. Recent experimental results showed already that Ge and Si nanowires can be used as high performance field-effect transistors. ${ }^{1}$ It was also found that these wires have a high potential as sensors for biological and chemical substances, ${ }^{2}$ and as logic gates. ${ }^{3}$ Photoluminescence of Ge wires was observed ${ }^{4}$ and Ge dot structures were found to be useful in efficient solar cells. 5

Previous theoretical research focused mainly on the electronic $^{6}$ and optical properties ${ }^{7,8}$ of freestanding Ge NWs and the effect of quantum confinement on these properties. Doped wires have not yet been theoretically explored, while it is well known that dopants can alter the electronic properties substantially. ${ }^{9}$ The focus of this letter will be the investigation of the properties of doped Ge nanowires. As dopants we consider boron ( $p$-type $\mathrm{NW}$ ) and phosphorus ( $n$-type NW). We study (i) the formation energy needed to create the doped wires, (ii) the preferred position of the dopants, and (iii) the changes that occur when a dangling bond is introduced. These results will be compared with recent $a b$ initio studies on doped Si nanowires. ${ }^{10}$

We consider hydrogen passivated wires, which can be obtained experimentally using the vapor-liquid-solid growth method or the oxide-assisted growth method. ${ }^{11-13}$ These wires grow dominantly along the [111] direction. ${ }^{14}$ Growth along the [110] direction is also observed. ${ }^{14,15}$ For our numerical study we considered wires in the [110] direction with diameters of 1.2 and $1.6 \mathrm{~nm}$, containing 28 and 62 atoms in a unit cell, respectively. These unit cells are shown in Fig. 1. Our calculations are performed within density-functional theory using the local density approximation for the exchange-correlation functional. The present results have been obtained through the use of the ABINIT code ${ }^{16}$ and Troullier-Martin ${ }^{17}$ pseudopotentials. A kinetic energy cutoff of 20 hartree was used for the undoped and P-doped Ge NWs, and of 30 hartree for the B-doped Ge NWs. A $1 \times 1$ $\times 4$ Monkhorst-Pack ${ }^{18}$ grid was used and all structures were relaxed without imposing any symmetry constraint. The structures are considered to be relaxed if the largest force

\footnotetext{
${ }^{a}$ Electronic mail: hartwin.peelaers@ua.ac.be

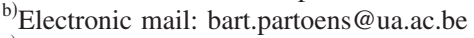

${ }^{c)}$ Electronic mail: francois.peeters@ua.ac.be
}

component is smaller than $5 \times 10^{-5}$ hartree/bohr. Due to the periodic boundary conditions, a layer of vacuum was used in the $x$ and $y$ directions to create an infinite one-dimensional system.

In order to study the preferential positions of the dopants in the wires, we calculated the formation and the segregation energies. The formation energy $E_{f}$ of an impurity (B and/or $\mathrm{P})$ is defined as the energy needed to insert an atom B and/or $P$ (taken from a reservoir) into the wire after removing one (or more) Ge atoms (to a reservoir, assumed to be bulk Ge),

$$
\begin{aligned}
E_{f}= & E(\text { doped Ge NW })-E(\mathrm{Ge} \mathrm{NW})+(n+m) \mu_{\mathrm{Ge}} \\
& -n \mu_{\mathrm{B}}-m \mu_{\mathrm{P}},
\end{aligned}
$$

where $n$ and $m$ are the numbers of, respectively, $\mathrm{B}$ and $\mathrm{P}$ dopants, $\mu_{\mathrm{Ge}}$ the chemical potential of Ge (here set equal to the total energy per atom of bulk Ge), and $\mu_{B / P}$ the chemical potential of the impurity atom. For these chemical potentials we use the total energy per atom in the tetragonal $B_{50}$ crystal for $\mathrm{B}$ and the orthorhombic black phosphorus for $\mathrm{P}$.

The segregation energy $E_{s}$ is defined as the energy difference between a Ge NW doped in the center and one doped at the edge of the wire,

$$
\begin{aligned}
E_{s}= & E(\text { centered doped Ge NW }) \\
& -E(\text { edge doped Ge NW }) .
\end{aligned}
$$

We will first study the most likely location of B and P dopants in these wires. This location can be found by comparing the formation energies. In Fig. 2 the formation energy is shown for the different possible locations in the $1.2 \mathrm{~nm}$ wire, for different distances (in the wire direction) between the
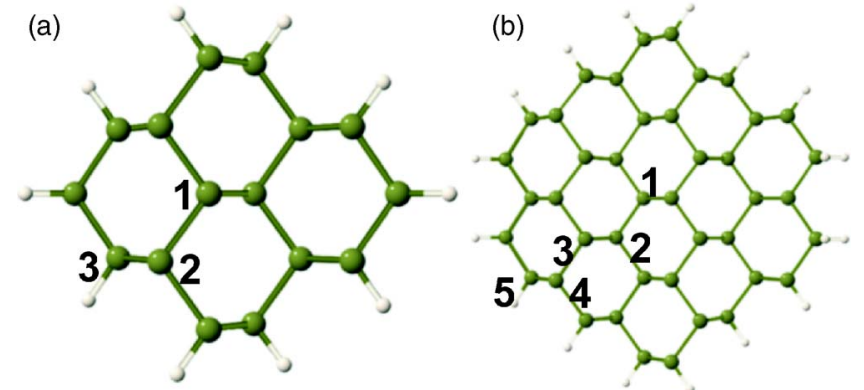

FIG. 1. (Color online) Relaxed unit cell of NW: (a) the $1.2 \mathrm{~nm}$ [110] wire consists of $16 \mathrm{Ge}$ and $12 \mathrm{H}$ atoms and (b) the $1.6 \mathrm{~nm}$ wire consists of $42 \mathrm{Ge}$ and $20 \mathrm{H}$ atoms. 


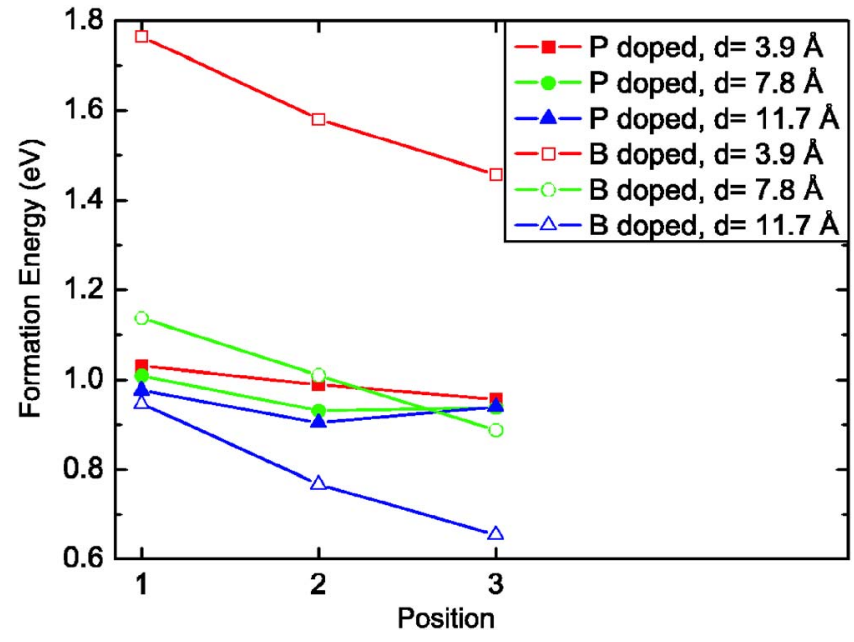

FIG. 2. (Color online) Formation energy of the $1.2 \mathrm{~nm}$ wire as a function of the dopant position [see Fig. 1(a)] and the dopant separation $d$ along the wire axes.

dopants (here 3.9, 7.8, and $11.7 \AA$ ). The positions are labeled as shown in Fig. 1(a). This figure shows that the formation energy decreases when a B dopant is located closer to the edge of the wire. The corresponding segregation energy is $0.3 \mathrm{eV}$. A similar trend is found for $\mathrm{P}$ dopants, but much smaller $\left(E_{s}=70 \mathrm{meV}\right)$. This effect occurs due to relaxation. Indeed the Ge cagelike structure (zinc blende) is more robust in the center regions, so that the amount of allowed relaxation is limited. For the impurity in the edge position the structure can be deformed more easily, which allows for an increased relaxation lowering the total energy. This is illustrated in Fig. 3, which shows the formation energy for B and $\mathrm{P}$ doped $1.2 \mathrm{~nm}$ nanowires for an unrelaxed structure (using the structure of the relaxed undoped wire as starting atom positions) and a fully relaxed structure. The effect of relaxation is much larger $(1.3 \mathrm{eV}$ for the center location and $1.7 \mathrm{eV}$ for the edge location) for $\mathrm{B}$ dopants than for $\mathrm{P}$ dopants $(0.17$ and $0.3 \mathrm{eV}$, respectively, for the center and the edge). This difference is related to the relative atom size: B atoms are smaller than $\mathrm{P}$ atoms, which are smaller than $\mathrm{Ge}$

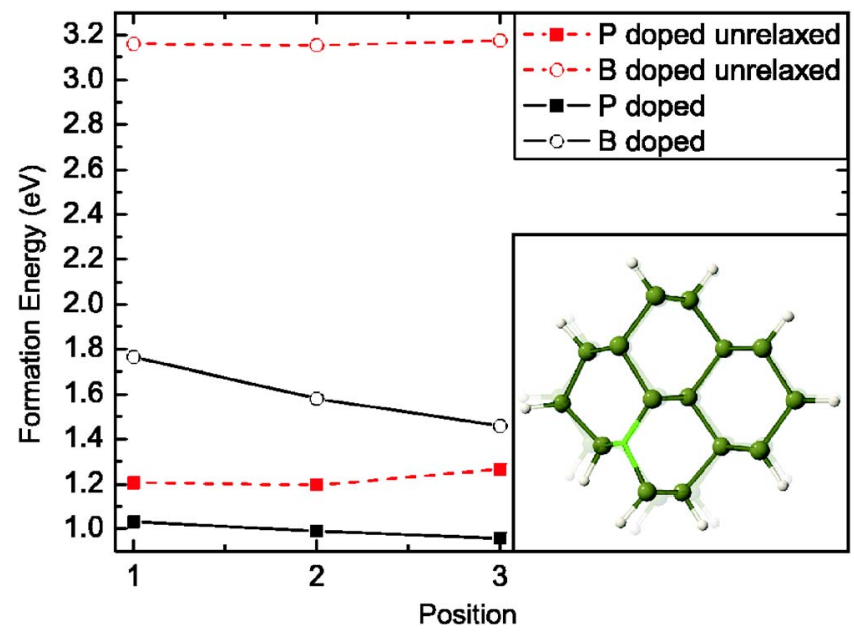

FIG. 3. (Color online) Formation energy of the smallest wire $(1.2 \mathrm{~nm})$ for relaxed and unrelaxed structures. The effect of the relaxation lowers the formation energy and this effect is larger for a P doped wire than it is for a $\mathrm{B}$ doped one. The effect is also larger for position 3 (the edge location). The inset shows the relaxed structured for a wire with a B dopant on position 2.

The transparent image shows the unrelaxed structure. creases, as was the case in the wires without DB, but the
Downloaded 26 Jun 2007 to 143.129.131.61. Redistribution subject to AIP license or copyright, see http://apl.aip.org/apl/copyright.jsp

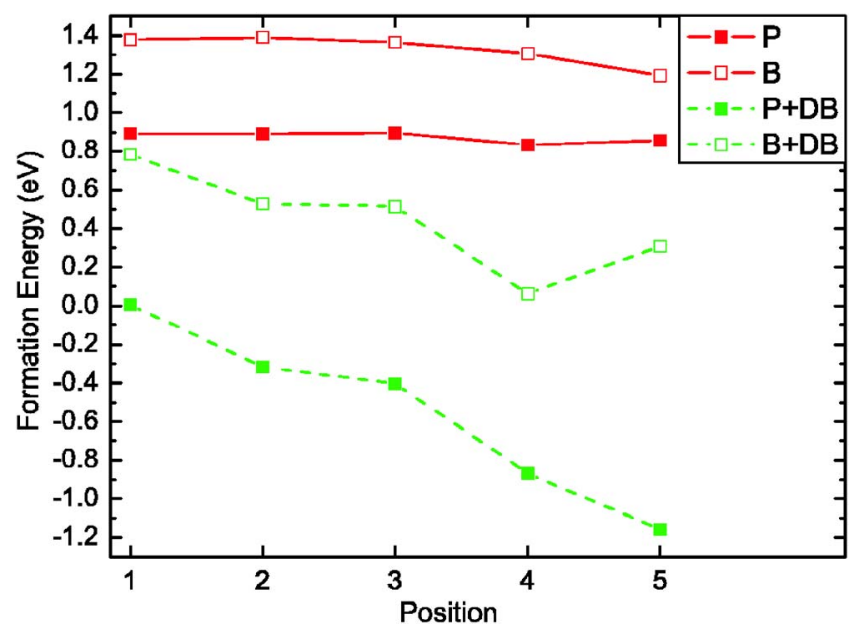

FIG. 4. (Color online) Formation energy for the $1.6 \mathrm{~nm}$ wire in the presence and absence of a dangling bond as a function of the dopant position [see Fig. $1(b)]$.

atoms. Thus B dopants can benefit more from lattice relaxation. When the distance in the wire direction between the dopants increases, the formation energy decreases because interactions between dopants also decrease with distance. Please note that, while the dopant concentrations are very high, this does not influence our conclusions because the trends are the same for all considered dopant concentrations. Figure 4 shows the formation energy for the different dopants in case of the thicker $(1.6 \mathrm{~nm})$ wire (full lines). The qualitative behavior is similar to that of the $1.2 \mathrm{~nm}$ wire.

Here we notice differences with equivalent Si wires. ${ }^{10}$ For Si the preferred position was always the near edge position (position 2 or 4 , respectively, for the 1.2 or $1.6 \mathrm{~nm}$ wires). For Si two competing interactions were observed when the axial distance between the dopants was increased: a repulsive and an attractive one (for very small distances), while for $\mathrm{Ge}$ there is only a repulsive interaction.

A dangling bond (DB) is a common defect occurring in hydrogenated nanowires. This defect occurs when one of the passivating hydrogen atoms is missing. A reasonable estimation for the concentration of these DB defects at a surface, based on this concentration for Si surfaces, is $10^{12} \mathrm{~cm}^{-2}$. ${ }^{19}$ When considering a typical dopant concentration of 5 $\times 10^{18} \mathrm{~cm}^{-3}$, the number of DB can be larger than the number of dopants for wires with a diameter smaller than $4 \mathrm{~nm}$. We will now investigate the effect that such a DB has on the preferred positions of the dopants. Therefore, we calculated the formation energies for the dopants at different positions in the presence of a missing hydrogen atom. The results of these calculations are shown for the $1.2 \mathrm{~nm}$ wire in Fig. 5 and for the $1.6 \mathrm{~nm}$ wire in Fig. 4 (dashed lines).

This figure shows that the effect of a DB is very pronounced for P dopants: the formation energy of these dopants is decreased considerably near the edge in comparison with the center position. The formation energy for B dopants is the lowest in a near edge position (position 2 in the $1.2 \mathrm{~nm}$ wire or position 4 in the $1.6 \mathrm{~nm}$ wire). As can be seen from the inset of Fig. 5, which shows the relaxed unit cell in case of a B dopant on position 2, the difference between position 2 and position 3 is very small, so we will use this position to calculate the segregation energy. When the dopant separation in the wire direction increases, the formation energy decreases, as was the case in the wires without DB, but the 


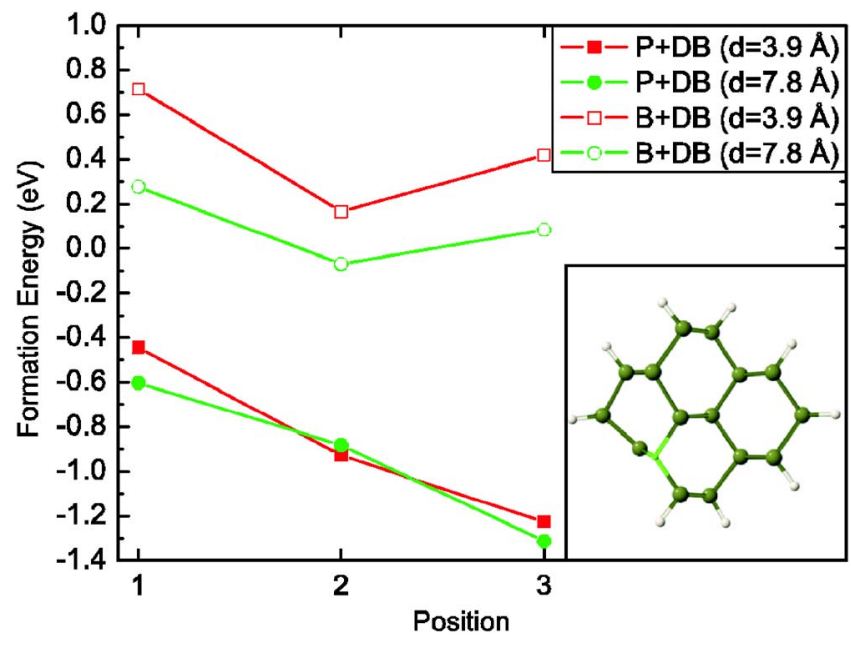

FIG. 5. (Color online) Formation energy of the $1.2 \mathrm{~nm}$ wire, in the presence of a DB defect, given as a function of the dopant position for two different dopant separations along the wire axis $(d=3.9$ and $7.8 \mathrm{~nm})$. The inset shows the relaxed unit cell for a B dopant placed at position 2 .

segregation energy remains of the same order of magnitude. The value for this segregation energy (for a dopant separation of $7.8 \AA$ ) for a P dopant is of the order of $1 \mathrm{eV}$, while for $\mathrm{B}$ dopants it is of the order of $0.5 \mathrm{eV}$. This indicates that for similar dopant concentrations the fraction of $\mathrm{P}$ dopants near the edge will be larger than the fraction of B dopants. When a dopant is located near the DB, this DB will "trap" the additional electron (for P) or hole (for B dopants), thus reducing the number of free carriers, and increasing the resistivity. This is similar to the behavior of $\mathrm{Si}$ nanowires, where this effect was calculated ${ }^{20}$ and measured. ${ }^{21,22}$

To conclude, we studied the localization of B and P dopants in Ge wires directed in the [110] direction. In the absence of dangling bond defects, B dopants prefer the edge positions, with a corresponding segregation energy of $0.3 \mathrm{eV}$, while this effect is much smaller for $\mathrm{P}$ dopants. This can be understood as due to a relaxation effect. When a dangling bond defect is introduced, this behavior changes: the segregation energy of $\mathrm{P}$ dopants increases to $1 \mathrm{eV}$. For $\mathrm{B}$ dopants the effect is less pronounced. Qualitatively similar behavior was found in Si wires.

This work was supported by the Flemish Science Foundation (FWO-Vl), the Belgian Science Policy (IAP), and TOP-BOF (University of Antwerp).

${ }^{1}$ Y. Cui, Z. Zhong, D. Wang, W. U. Wang, and C. M. Lieber, Nano Lett. 3, 149 (2003).

${ }^{2}$ Y. Cui, Q. Wei, H. Park, and C. M. Lieber, Science 293, 1289 (2001).

${ }^{3}$ Y. Huang, X. Duan, Y. Cui, L. J. Lauhon, K.-H. Kim, and C. M. Lieber, Science 294, 1313 (2001).

${ }^{4}$ M. Halsall, H. Omi, and T. Ogino, Appl. Phys. Lett. 81, 2448 (2002).

${ }^{5}$ A. Alguno, N. Usami, T. Ujihara, K. Fujiwara, G. Sazaki, and Y. S. K. Nakajima, Appl. Phys. Lett. 83, 1258 (2003).

${ }^{6}$ R. Kagimura, R. Nunes, and H. Chacham, Phys. Rev. Lett. 95, 115502 (2005).

${ }^{7}$ M. Bruno, M. Palummo, A. Marini, R. D. Solo, V. Olevano, A. Kholod, and S. Ossicini, Phys. Rev. B 72, 153310 (2005).

${ }^{8}$ S. Beckman, J. Han, and J. R. Chelikowsky, Phys. Rev. B 74, 165314 (2006).

${ }^{9}$ E. Tutuc, J. Chu, J. Ott, and S. Guha, Appl. Phys. Lett. 89, 263101 (2006).

${ }^{10}$ H. Peelaers, B. Partoens, and F. M. Peeters, Nano Lett. 6, 2781 (2006).

${ }^{11}$ A. M. Morales and C. M. Lieber, Science 279, 208 (1998).

${ }^{12}$ Y. Cui, L. J. Lauhon, M. S. Gudiksen, J. Wang, and C. M. Lieber, Appl. Phys. Lett. 78, 2214 (2001).

${ }^{13}$ N. Wang, Y. H. Tang, Y. F. Zhang, C. S. Lee, I. Bello, and S. T. Lee, Chem. Phys. Lett. 299, 237 (1999).

${ }^{14}$ H. Jagannathan, M. Deal, Y. Nishi, J. Woodruff, C. Chidsey, and P. C. McIntyre, J. Appl. Phys. 100, 024318 (2006).

${ }^{15}$ D. Wang and H. Dai, Angew. Chem., Int. Ed. 41, 4783 (2002).

${ }^{16}$ X. Gonze, J.-M. Beuken, R. Caracas, F. Detraux, M. Fuchs, G.-M. Rignanese, L. Sindic, M. Verstraete, G. Zerah, F. Jollet, M. Torrent, A. Roy, M. Mikami, Ph. Ghosez, J.-Y. Raty, and D. C. Allen, Comput. Mater. Sci. 25, 478 (2002).

${ }^{17}$ N. Troullier and J. L. Martins, Phys. Rev. B 43, 1993 (1991).

${ }^{18}$ H. J. Monkhorst and J. D. Pack, Phys. Rev. B 13, 5188 (1976).

${ }^{19}$ A. Stesmans and V. V. Afanas'ev, J. Phys.: Condens. Matter 10, L19 (1998).

${ }^{20}$ M. V. Fernández-Serra, C. Adessi, and X. Blase, Phys. Rev. Lett. 96, 166805 (2006)

${ }^{21}$ Y. Cui, X. Duan, J. Hu, and C. M. Lieber, J. Phys. Chem. B 104, 5213 (2000).

${ }^{22}$ J.-Y. Yu, S.-W. Chung, and J. R. Heath, J. Phys. Chem. B 104, 11864 (2000). 